\title{
Test method and device for evaluation of volatile corrosion inhibitors
}

\author{
M. Sieber,* S. Lautner and F. Faßbender \\ ${ }^{1}$ EXCOR Korrosionsforschung GmbH, Magdeburger Straße 258, D-01067 Dresden, \\ Germany \\ *E-mail: maximilian.sieber@excor.de
}

\begin{abstract}
Volatile corrosion inhibitors (VCI), which are incorporated in packaging materials like films, papers, plastic racks etc., are a suitable means for temporary corrosion protection during transport and storage of metallic products. For the evaluation of the corrosion protection effect of VCI-containing products, numerous tests are established. In most cases, principally, a metallic sample is exposed to a climatic stress in presence of a VCI-containing product and the protection effect of the VCI is evaluated afterwards by visually assessing the occurrence/absence of corrosion products at the sample surface. In a newly proposed test method and a dedicated device, the evaluation of the VCI-containing product is based on electrochemical measurements at the interface between the metallic sample and a film of condensed water. The water film is precipitated during the test by actively cooling the metallic sample below the dew point, which imposes a corrosive stress on the sample surface. With a simple direct current potentiostat for polarization resistance measurement, the state of the metallic surface (corrosion-active, passive) is registered objectively during the test and thus the corrosion protection effect of the VCI is evaluated. In the present paper, the test method and the dedicated device are disclosed in detail. On the basis of a statistical analysis of a sample of independently repeated tests, limit values are established for the quality assessment of VCI products. Different commercially available VCI films are evaluated and the results are compared to the state-of-the-art jar test procedure based on German TL 8135. In conclusion, the proposed test reproducibly provides objective and quantitative information on the corrosion protection effect of VCI-containing products.
\end{abstract}

Keywords: volatile corrosion inhibitors, atmospheric corrosion testing, polarization resistance.

Received: July 24, 2019. Published: October 25, 2019

doi: $\underline{10.17675 / 2305-6894-2019-8-4-7}$

\section{Introduction}

Bare metal surfaces of any type of goods as well as semi-finished products are prone to corrosion not only during service, but already during transportation and storage. Temperature changes and the omnipresent humidity in the atmosphere can impose severe corrosive stress. Especially for surfaces of iron-based materials, which are not already adequately protected by permanent coatings, appropriate temporary corrosion protection is 
necessary. A temporary coating based on organic solvents, water, oil or wax can keep corrosive media away from the metallic surfaces, but involves costly operations for application, removal and disposal. A desiccant within a packaging decreases the humidity of the atmosphere around a product, but requires a technically impermeable outer packaging, which also must not be opened during transportation and storage (e.g. during customs clearance or partial unpacking). The corrosion protection by volatile corrosion inhibitors (VCI, also denoted as vapor phase corrosion inhibitors - VPCI) is based on the release of inhibitors from films, papers, cardboards, plastic racks, diffusers or oils. The inhibitors diffuse within the packaging, interact with the metallic surface, strengthen the primary oxide layer and thus impede corrosion. The inhibitors rapidly volatilize after unpacking. VCI thus allow for immediate use of the goods after transportation and storage without additional cleaning operations and provide corrosion protection up to several years even if the outer packaging is occasionally opened. Detailed information on the VCI method as well as on current developments are given in [1-7].

To evaluate the corrosion protection effect of VCI-containing products for quality control or research and development purposes, numerous tests have been established. In general practice, a metallic sample is exposed to a climatic stress in presence of a VCIcontaining product and the protection effect of the VCI is evaluated after the test by visually assessing the occurrence or absence of corrosion products on the metallic sample. The climatic stress during service is usually simulated by altering the temperature and the humidity of the atmosphere in the course of the test. Thus, results on the corrosion protection ability of VCI are provided on a phenomenological basis. The major drawback of this approach is that the evaluation is prone to errors and deviation, since the visual assessment of the metallic surface after the test is often subjective and rarely automatized. Recognition of corrosion products by digital image processing is elaborate, especially in terms of calibration. Further, any test carried out in a climate chamber utilizing alternating temperature regimes is only fully comparable for metallic samples of equal mass, because the thermodynamic inertia of the metallic sample is a ruling factor for condensation of water on the sample and thus for the corrosive load. For VCI-carrying products, which absorb large amounts of water, for instance papers or cardboards, the humidity in a test volume may be decreased significantly in comparison to polymer-based carriers like films, which hardly absorb humidity. This also results in a different corrosive load for different carrier materials. Moreover, to gain information on the point of time of the corrosion initiation, excessive parallel sampling is necessary since most of the established tests do not allow for interim observation of the metallic samples and continuation of the test afterwards.

In a newly proposed test method and a dedicated test device derived from previous research covered in [8], the mentioned drawbacks are addressed. A metallic sample is exposed in a small test chamber alongside a VCI-containing product. After a defined amount of time, during which the corrosion inhibitors volatilize, reach the metallic sample and there interact with the primary oxide layer, a corrosive stress is applied by humidifying 
the atmosphere in the chamber and by cooling down the sample below the dew point via a Peltier element. Consequently, a film of condensed water precipitates on the metallic sample. Electrochemical measurements are then carried out to characterize the interface between the metallic sample and the condensed water film.

In contrast to state-of-the-art testing procedures for VCI-containing products, the climatic stress imposed via a Peltier element is neither affected by the mass of the metallic sample, because its temperature is actively regulated with reference to the dew point, nor is it significantly altered by different water absorption behavior of different VCI-carriers. Moreover, the electrochemical measurement allows for both objective and time-resolved characterization of the surface state (passivity or corrosion-activity). In this respect, numerous studies have established correlations between values obtained from electrochemical measurements in direct current mode or in alternating current mode and the passivity/inhibition or corrosion activity of a metallic surface [9-16]. In any case, the basis for the evaluation is the relation of current density and potential. Passivity is tantamount to low electrochemical activity, which means that, for instance, a perturbation of the potential leads only to a very small increase of the current density. In contrast, high electrochemical activity leads to a significant current flow in case of potential perturbation. Regarding the conditions of the considered tests, this indicates corrosion of the metallic sample. Nevertheless, it has to be noted that the formation of a passive film is also accompanied by high electrochemical activity. This is, however, limited to a short period of time at the beginning of the exposition of the metallic sample alongside the VCI material in the test atmosphere and is therefore a non-equilibrium state phenomenon.

Hereinafter, the proposed test procedure and the dedicated device will be disclosed in detail. Limit values are established, which allow for quality assessment of the corrosion protection effect of VCI-containing products based on the measurement of the polarization resistance. The corrosion protection effect of different VCI-films is evaluated in the newly proposed test and compared to results of the state-of-the-art jar test procedure, for reference.

\section{Experimental}

\subsection{Sample material and test arrangement}

Metallic samples with a diameter of $16 \mathrm{~mm}$ and a height of approx. $10 \mathrm{~mm}$ were made from steel 235JRG. The sample material has the following nominal composition: $\mathrm{C}<0.2$ wt. $\%, \mathrm{Mn}<1.4$ wt. $\%, \mathrm{P}<0.045$ wt. $\%$, Fe balance. The samples were prepared by grinding the upper surface of the cylindric samples with SiC-paper (600 grit), rinsing with deionized water, rinsing with ethanol and drying under warm air. Afterwards, the samples were stored in a desiccator for at least 3 days. 4 commercially available VCI-doped polyethylene-films of different manufacturers were used to demonstrate the evaluation of the corrosion protection ability with the proposed test method and device. The VCI-films were chosen on the basis of preliminarily performed jar tests (see sections 2.3 and 3 ) in 
order to represent a wide range of corrosion protection ability with respect to the used steel substrate. So, the plausibility of the new test method can be checked. Also, the ability of the test method to distinguish between different VCI-films was addressed by the chosen VCI-films. Therefore, it is noticeable that there were commercially available products among the chosen VCI-films, which are not specifically dedicated to corrosion protection of iron-based substrate materials. For reasons of fairness among different and suppliers of VCI-products and for reasons of confidentiality, the VCI-films are denoted anonymously as VCI-film I, II, III and IV. Additionally, all the tests were carried out without any VCImaterial. The test arrangement is schematically shown in Figure 1 (apparatus) and Figure 2 (sample holder).

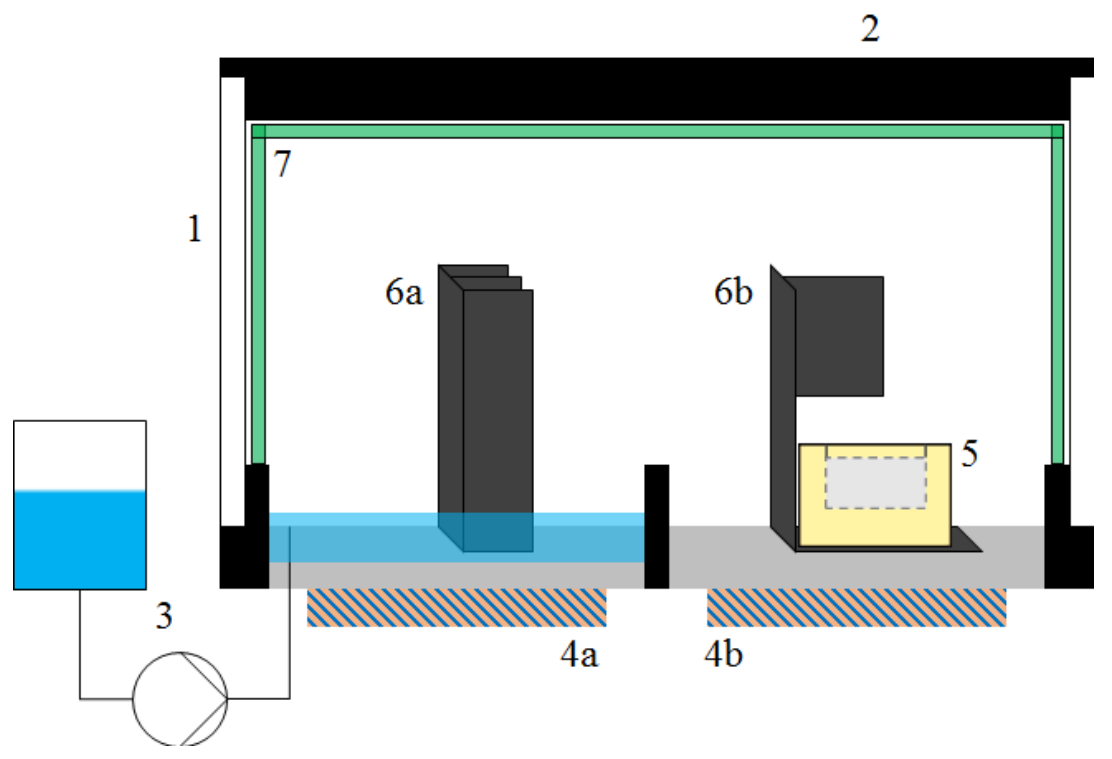

Figure 1. Principal test arrangement with PMMA cylindrical tube (1), POM lid (2), water reservoir with pump (3), two separate Peltier elements mounted below aluminum plates (4), sample holder with sample (5), two fin-shaped aluminum heatsinks (6) and VCI-film (7) [17].

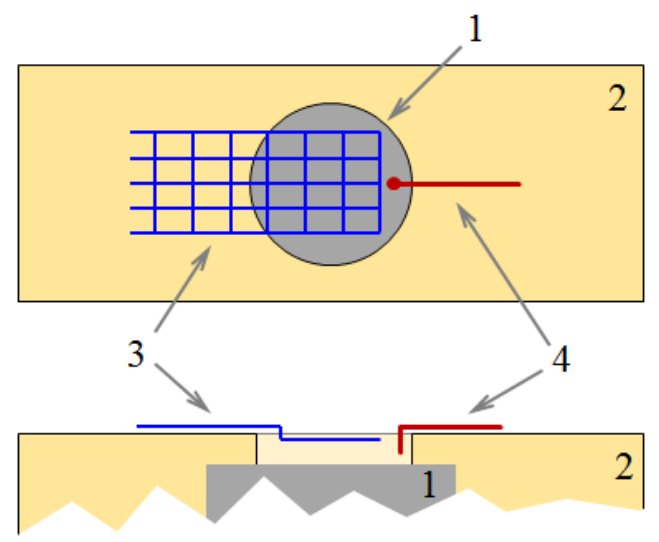

Figure 2. Principal layout of the sample holder (2) with sample (1) and auxiliary electrodes, namely a platinized titanium mesh (3) and a platinum wire (4) acting as counter and reference electrode, respectively [17]. 
The test setup consisted of a cylindrical test chamber. The chamber was made from poly(methyl methacrylate) (PMMA-tube) and polyoxymethylene (POM-lid). The volume of the chamber was approx. 4.5 l. Temperature and relative humidity in the chamber were monitored and the dew point was calculated from this data, continuously. The VCI film was brought into the chamber so that both the inside of the cylindrical tube and of the lid were covered. The bottom of the chamber consisted of 2 aluminum plates, which were insulated thermally and electrically by POM. A separate Peltier element was mounted to each of the aluminum plates. One of the plates formed a reservoir, in which water was injected during the course of the test by a peristaltic pump. By heating of the aluminum plate under the water reservoir via the attached Peltier element, water was evaporated and thus the atmosphere in the chamber was humidified. Also, an extruded fin-shaped heatsink was mounted on the aluminum plate. Thus, by cooling of the plate via the Peltier element, humidity condensed on the fin-shaped heatsink and the atmosphere in the chamber was dehumidified. The temperature of the aluminum plate was monitored by a type $\mathrm{K}$ thermocouple sensor attached to the plate.

On the other aluminum plate, a sample holder was placed, which contained the prepared steel sample. The sample holder consisted of polyamide PA12, which was epoxysealed. The sample holder had an aperture, under which the ground surface of the metallic sample was positioned. A ring seal was inserted between said surface and the sample holder and thus the active sample surface was defined. Above the aperture, a platinized titanium mesh was positioned in a distance of approx. $2 \mathrm{~mm}$ to the sample surface. The mesh served as counter electrode for the electrochemical measurement. A platinum wire was also positioned above the aperture so that its tip had a distance of approx. $1 \mathrm{~mm}$ to the sample surface. The wire served as a pseudo-reference electrode during the measurement. The pseudo-reference electrode was chosen to avoid contamination of the system by the use of conventional reference electrodes containing corrosion-promoting ions like chloride, sulfate or the like. The sample holder was such that the sample was thermally connected to the aluminum plate and thus to the Peltier element. By cooling via the Peltier element and controlling the sample temperature by a type $\mathrm{K}$ thermocouple sensor, the sample temperature could be set below the dew point of the atmosphere in the chamber so that humidity condensed on the sample surface. This corresponds to the condensation of water, which typically occurs during transportation during night/day cycle or when cold parts are brought into a heated room from the outside. Additionally, an auxiliary profile made from extruded, enameled aluminum was placed above the aperture of the sample holder in a distance of approx. $10 \mathrm{~mm}$. The profile was thermally connected to the same aluminum plate as the sample and thus to the Peltier element. While humidity condensed on the sample, it would therefore also condense on the auxiliary profile. From there, a quantity of condensed humidity would drop on to the sample. This simulates so-called container sweat, which typically forms during transportation when the whole packaging and its included atmosphere is cooled down from higher to lower temperatures and moisture precipitates. In that case, the contained humidity condenses inside the packaging and drops 
onto the packaged goods. After the end of the test, the metallic sample was dried outside the chamber under a stream of warm air [17].

\subsection{Test regime, electrochemical measurements}

The requirements with regard to the test regime were as follows: (1) simulate critical climatic stress imposed on metallic goods during transportation, (2) ensure wetting of sample surface and auxiliary electrodes to allow for electrochemical measurement during some state of the test, (3) impose sufficient stress to significantly corrode the sample in absence of any appropriate VCI material, (4) allow for differentiation of VCI-containing products with regard to their corrosion protection effect, (5) allow for short-term examination of the corrosion protection effect of VCI-containing products (preferably within 1 working day). The test regime as well as the principal layout of the apparatus to realize the test regime, which is disclosed in section 2.1 , have been the subject of extensive preliminary investigation.

The principal course of the test with regard to the conditions in the test chamber is shown in Figure 3. At the beginning of the test, the chamber as well as the aluminum plates are at room temperature. In the first stage of the test $(2 \mathrm{~h})$, both the Peltier elements are kept in passive state, i.e. their temperature is not regulated. Water is pumped into the chamber and humidifies the atmosphere by free evaporation. Meanwhile, inhibitors are set free from the VCI-containing product and may diffuse in the chamber and reach the metallic sample surface. In Stage 2, a short corrosive load is applied. The sample in the sample holder is cooled down $3 \mathrm{~K}$ below the dew point for $15 \mathrm{~min}$. A small amount of humidity condenses on the surface. The short duration of the cooling phase is neither sufficient to wet all electrodes nor does additional condensed humidity drop on the sample from the auxiliary aluminum profile, which is thermally connected to the sample. The metallic sample is afterwards heated $5 \mathrm{~K}$ above the dew point for $15 \mathrm{~min}$. Meanwhile, the water reservoir is emptied and the corresponding aluminum plate with the fin-shaped heat sink is cooled down to $10^{\circ} \mathrm{C}$ so that the air in the chamber is dehumidified to ensure that the small amount of condensed humidity on the metallic sample evaporates quickly and completely. So, the sample surface is dried. Stage 2 thus simulates a mild corrosive stress by short-time condensation of humidity and subsequent drying and is carried out thrice. In Stage 3 of the test, the Peltier elements are in passive state again. Again, water is injected into the reservoir in the chamber and humidifies the atmosphere. Afterwards, stage 4 is entered, during which the main corrosive stress is imposed. Therefore, the aluminum plate below the filled water reservoir is heated to $60^{\circ} \mathrm{C}$, which leads to excessive evaporation and thus oversaturation of the atmosphere in the chamber with humidity. In the same time, the metallic sample and the attached auxiliary aluminum profile are cooled down $7 \mathrm{~K}$ below the dew point. This leads to severe condensation both on the metallic sample and the auxiliary profile. After approximately $30 \mathrm{~min}$, condensed humidity on the auxiliary profile starts to drop on to the metallic sample. After approximately $45 \mathrm{~min}$ in stage 4, the metallic sample surface as well as the auxiliary electrodes are sufficiently wetted to allow for 
electrochemical measurements. Because of the heating of the water reservoir, the atmosphere in the chamber is also heated and reaches a temperature of approx. $35^{\circ} \mathrm{C}$. The corrosive stress in this stage originates from the condensation of humidity directly on the sample surface, which is below the dew point, and the condensed water dropping on the surface from the auxiliary fin-shaped profile ( $c f$. container sweat).

In the standard procedure, the test is terminated after approx. $60 \mathrm{~min}$ in stage 4 . This equals a total test duration of approx. $7 \mathrm{~h}$. The corrosion protection ability of the VCIcontaining product is assessed on the basis of the measured polarization resistance at this point of time. The described procedure is used for quality assessment of VCI-containing products on the basis of an electrochemical measurement under the film humidity, which was condensed from the atmosphere before.

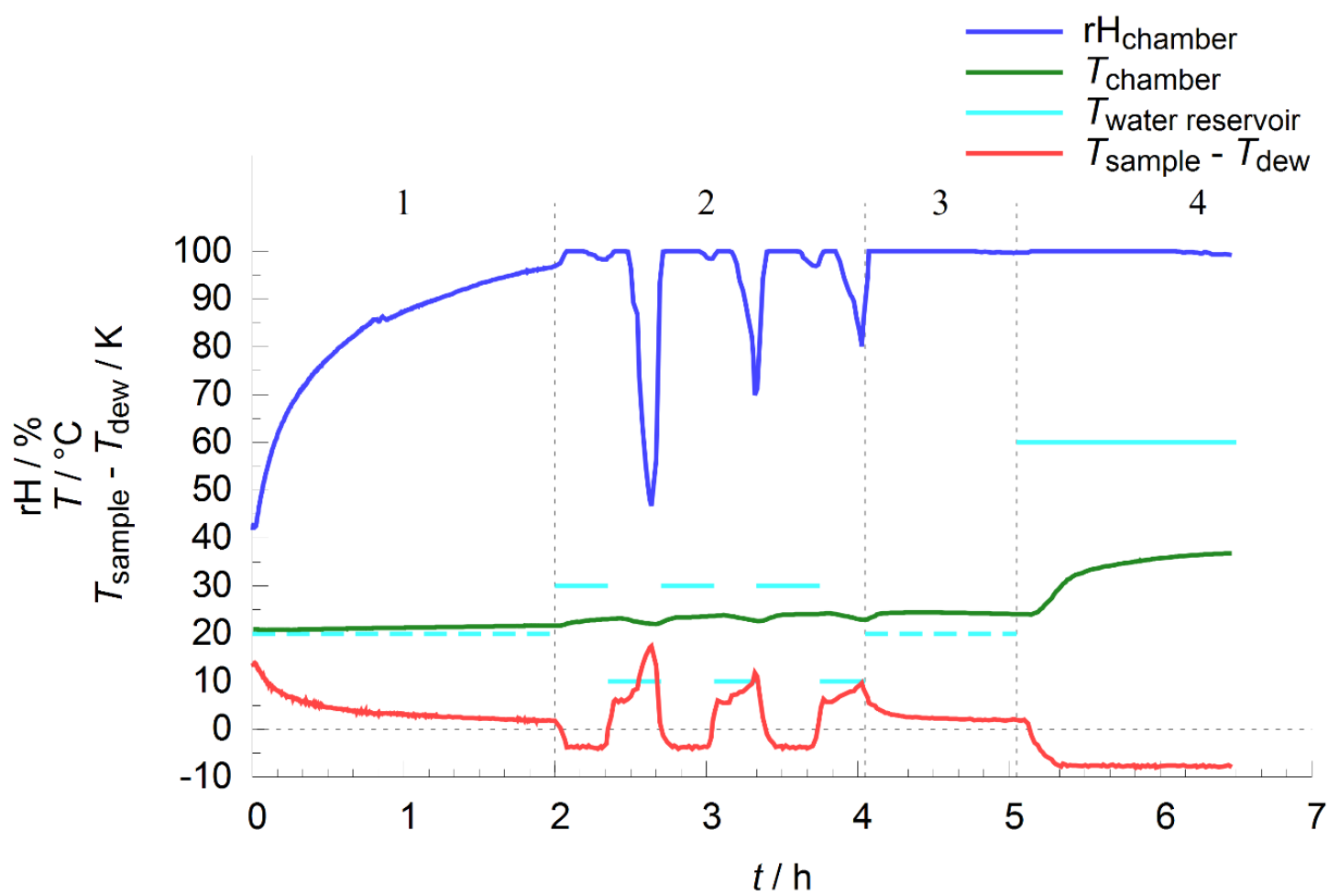

Figure 3. Evolution of relative humidity and temperature in the test chamber and of the sample temperature with respect to the dew point over the course of the test. Additionally, the set temperature for the water reservoir in the chamber is plotted (dashed line: Peltier element below the water reservoir is in passive state, i.e. no temperature regulation). Corrosive stress is applied on the metallic sample in test stages 2 and 4, when the sample is cooled below the dew point of the atmosphere in the chamber [17].

Electrochemical measurements were carried out with a workstation IM6 (Zahner, Germany). The elements of the 3-electrode-arrangement are described above ( $c f$. Figure 2). The use of the platinum wire pseudo-reference electrode did not allow for an exact determination of the electrochemical potential of the sample. However, the determination of the polarization resistance of the system by a small-range potential-sweep does not 
require knowledge of the absolute potential, but is based on the evaluation of the change of current density relative to the change of the potential. Polarization resistance measurements were carried out by applying a polarization of $0-9 \mathrm{mV}$ (scan rate: $1 \mathrm{mV} / \mathrm{s}$ ) relative to the equilibrium potential against the pseudo-reference electrode (open circuit). The small polarization avoids sample corrosion due to the measurement. In the mentioned potential range, the current-density-potential-behavior is approximately linear, so that the polarization resistance is obtained by determination of the mean inverse slope of the recorded curve in the measured range.

\subsection{Jar test}

For comparison, the corrosion protection effect of the 4 VCI-films was determined in a state-of-the-art jar test based on German TL 8135. The steel specimens used for the jar tests and the specimen used in the newly proposed test were from the same material batch (section 2.1). The test arrangement is shown in Figure 4, a detailed description of the test is given in [18].

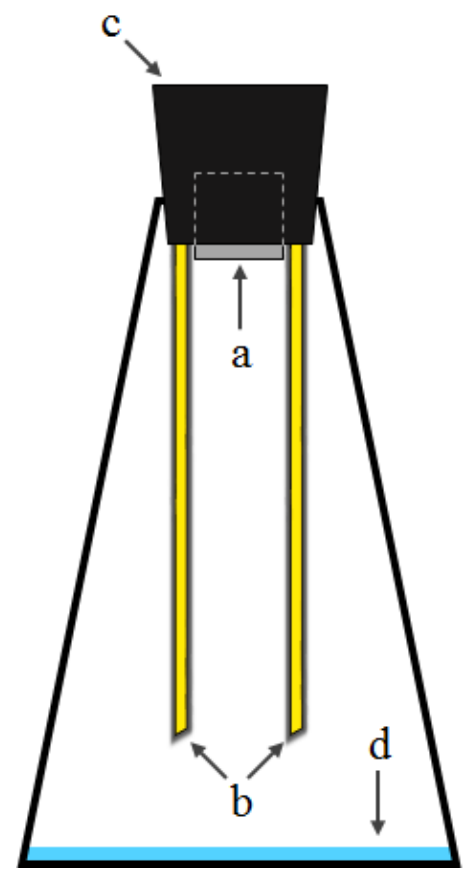

Figure 4. Principal arrangement of the jar test (volume of jar: 11 ) with ground metallic sample (a) and 2 stripes of the VCI-film with an overall active surface of $150 \mathrm{~cm}^{2}$ (b), which are mounted in a rubber plug (c). After $20 \mathrm{~h}$ of arid conditioning of the metallic sample in presence of the VCI-film at room temperature, a glycerol-water mixture (d) is added to the jar. After another $2 \mathrm{~h}$ of conditioning in the humidified atmosphere, the jar is put in a pre-heated cabinet at $40{ }^{\circ} \mathrm{C}$ for $2 \mathrm{~h}$ and afterwards, the corrosion protection effect of the VCI- film is evaluated based on the surface appearance of the metallic sample (a). [18] 


\section{Results and discussion}

\subsection{Corrosion protection effect of VCI-films in the jar test}

The results of the jar tests with the VCI-films are shown in Table 1. For reference, Figure 5 representatively shows the visual appearance of the surface of a steel specimen for each of the evaluation ranks. Based on the visual appearance of the steel specimens after the jar tests, VCI-films I and II offered good corrosion protection (no corrosion visible with unaided eye for most of the samples), while VCI-film III gives moderate corrosion protection (few smaller corrosion spots) in most cases. VCI-film IV has only weak to moderate corrosion protection ability (few smaller to some corrosion spots). As expected, the jar tests conducted without VCI-film resulted in no corrosion protection and a severely corroded surface of the steel specimens. It is noticeable that the jar test results fluctuate especially when VCI-films provide weak to moderate corrosion protection, while the results are more stable at both ends of the evaluation scale.

Table 1. Frequency of occurrence of the corrosion protection effect evaluations of the investigated VCIfilms in the jar test (3-good corrosion protection, 2 - moderate corrosion protection, 1 - weak corrosion protection, 0 - no corrosion protection).

\begin{tabular}{ccccccc}
\hline VCI-film & $\begin{array}{c}\text { No. of } \\
\text { samples }\end{array}$ & $\mathbf{0}$ & $\mathbf{1}$ & $\mathbf{2}$ & $\mathbf{3}$ & $\begin{array}{c}\text { Frequency of evaluation } \\
\text { Overall corrosion } \\
\text { protection }\end{array}$ \\
\hline I & 4 & - & - & 1 & 3 & $\operatorname{good}(3)$ \\
II & 22 & - & - & 4 & 18 & $\operatorname{good}(3)$ \\
III & 27 & 3 & 4 & 14 & 6 & moderate (2) \\
IV & 7 & 1 & 3 & 2 & 1 & weak to moderate (1-2) \\
without & 15 & 15 & - & - & - & no (0) \\
\hline
\end{tabular}
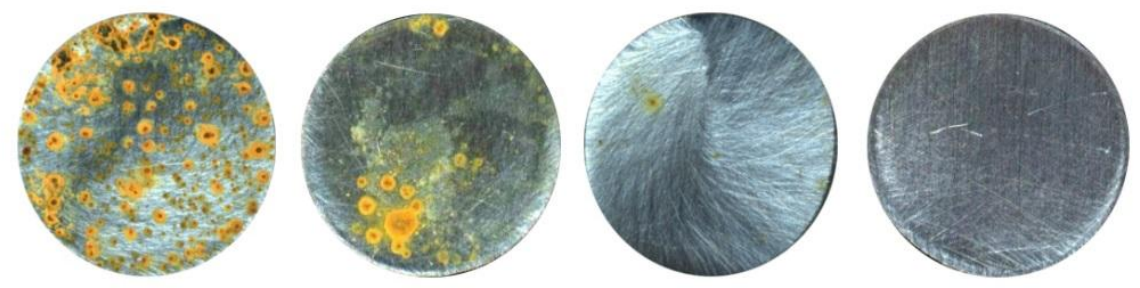

Figure 5. Representative visual appearance of steel specimens after the jar test from left to right: no corrosion protection (0), weak corrosion protection (1), moderate corrosion protection (2), good corrosion protection (3). 


\subsection{Reproducibility of corrosive load in the proposed test and evaluation scheme}

As described in section 2.2, in the newly proposed test the corrosive load is imposed by condensation and evaporation of humidity on and from the metallic sample surface, respectively. This is realized by actively cooling and heating the metallic sample below and above the dew point of the atmosphere in the test chamber. To ensure the reproducibility of the corrosive load, a reproducible regulation of the metallic sample's temperature with respect to the dew point is required. Figure 6 shows the course of the sample temperature with respect to the dew point for a plethora of tests, which were carried out independently. The focus is on the transition from stage 1 to stage 2 of the test (see section 2.2). In stage 1, no active regulation of the sample temperature is imposed and the sample itself is on room temperature. At the end of stage 1, the atmosphere in the chamber is already significantly humidified, so that the dew point is only $2-5 \mathrm{~K}$ below room temperature and therefore slightly below the unregulated sample temperature. However, the metallic sample remains completely dry throughout this first stage of the test (no corrosive load). From $t=2 \mathrm{~h}$ on, the corrosive load is imposed. Henceforth, the sample temperature is actively regulated. For all the experiments, the deviation of the sample temperature with respect to the dew point is minimal during the following cooling and heating cycle. For a set value of $3 \mathrm{~K}$ below dew point, the sample temperature actually is $3 \mathrm{~K}$ to $4 \mathrm{~K}$ below dew point. However, for all the considered tests, the absolute deviation of the sample temperature is below $1 \mathrm{~K}$ at any time during the cooling cycle. During the following heating phase, the humidity on the sample surface, which condensed during the previous cooling phase, is evaporated. The sample temperature is therefore set to $5 \mathrm{~K}$ above the dew point. The absolute deviation with regard to time for the transition from the cooling cycle to the heating cycle is $<2 \mathrm{~min}$. For a set temperature of $5 \mathrm{~K}$ above the dew point, the sample temperature actually reaches approx. $6 \mathrm{~K}$ above dew point with an absolute deviation of all the considered measurements of less than $1.5 \mathrm{~K}$. The described deviations are rather small and therefore present a good basis for a reproducible imposition of the corrosive load.

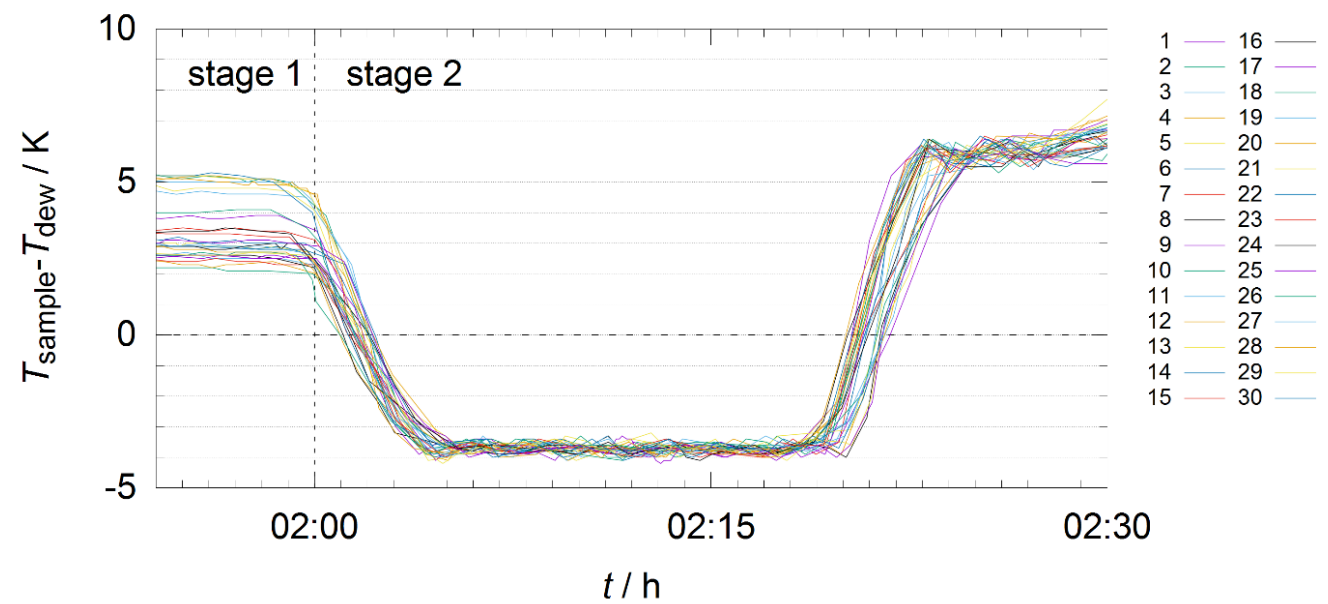

Figure 6. Evolution of the temperature of the metallic sample with respect to the dew point over the course of the first two stages of the test (conditioning $0-2 \mathrm{~h}$, mild corrosive stress $2-4 \mathrm{~h}$ ). 
Finally, the reproducibility is proven by a low deviation of the electrochemical values, which characterize the metallic sample's surface state (corrosion-activity/passivity) at the end of the test. Therefore, the test was independently repeated without any VCI-material for 10 times. For all the statistical operations, the polarization resistance has been transformed to the decadic logarithmic scale. A significant change of the corrosion protection effect is typically reflected by a change of the polarization resistance by orders of magnitude. Therefore, by consideration of confidence intervals in the linear scale, the effect and likeliness of deviation of the results is potentially underestimated for lower values (weak corrosion protection effect), while it is overestimated for higher values (high corrosion protection effect). Thus, the logarithmized values of the polarization resistance $R$ in $\Omega \cdot \mathrm{cm}^{2}$ were used as the basis for the calculation of the means (Equation 1) and the sample standard deviations (Equation 2) with $n$ - number of scores in the sample:

$$
\begin{gathered}
\bar{R}_{\log }=\frac{1}{n} \sum_{i=1}^{n} \log _{10} R_{i} \\
\sigma_{\log }=\sqrt{\frac{\sum_{i=1}^{n}\left(\log _{10} R_{i}-\bar{R}_{\log }\right)^{2}}{n-1}}
\end{gathered}
$$

For clarity, the given values for the means (Equation 3) and the $\sigma$-interval boundaries (equation 4) are delogarithmized:

$$
\begin{gathered}
\bar{R}^{*}=10^{\wedge} \bar{R}_{\log } \\
(\bar{R} \pm i \sigma)^{*}=10^{\wedge}\left(\bar{R}_{\log } \pm i \sigma_{\log }\right) \text { with } i=1,2,3, \ldots
\end{gathered}
$$

For tests without any VCI, Figure 7 depicts the results, the corresponding mean values

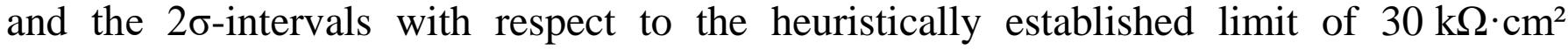
(approx. 4.5 on logarithmic scale), below which a significant corrosion attack takes place on the active surface of the metallic sample [17]. In contrast, values of the polarization resistance above $30 \mathrm{k} \Omega \cdot \mathrm{cm}^{2}$ indicate a good corrosion protection effect. For the tests without any VCI, the mean polarization resistance is 3.61 on the logarithmic scale (approx. $4.1 \mathrm{k} \Omega \cdot \mathrm{cm}^{2}$ ) with a sample standard deviation of 0.18 . The distance of the heuristically established limit for good corrosion protection to the mean of the sample without VCI is therefore $5 \sigma$. The $\pm 5 \sigma$ interval covers $99.99994 \%$ of the population. The probability that the polarization resistance exceeds the established limit in a test without VCI by chance is $<3 \cdot 10^{-5} \%$ (half of the remainder) and is thus marginal. With reference to the standard deviation of the polarization resistance, the large distance between the mean value without any VCI, which means without any corrosion protection, and the established limit for good corrosion protection justifies the introduction of another rating of the corrosion protection effect. Considering the shown sample of tests without VCI, the $2 \sigma$-interval in the logarithmic scale covers values from 3.25 to 3.97 (approx. $2 \mathrm{k} \Omega \cdot \mathrm{cm}^{2}$ to $9 \mathrm{k} \Omega \cdot \mathrm{cm}^{2}$ ). The 
probability that the polarization resistance exceeds the upper value of the $2 \sigma$-interval is approx. $2.3 \%$. Therefore, the upper value of the $2 \sigma$-interval of the test sample without any $\mathrm{VCI}$ is proposed as the limit to distinguish between an insufficient and a moderate corrosion protection effect.

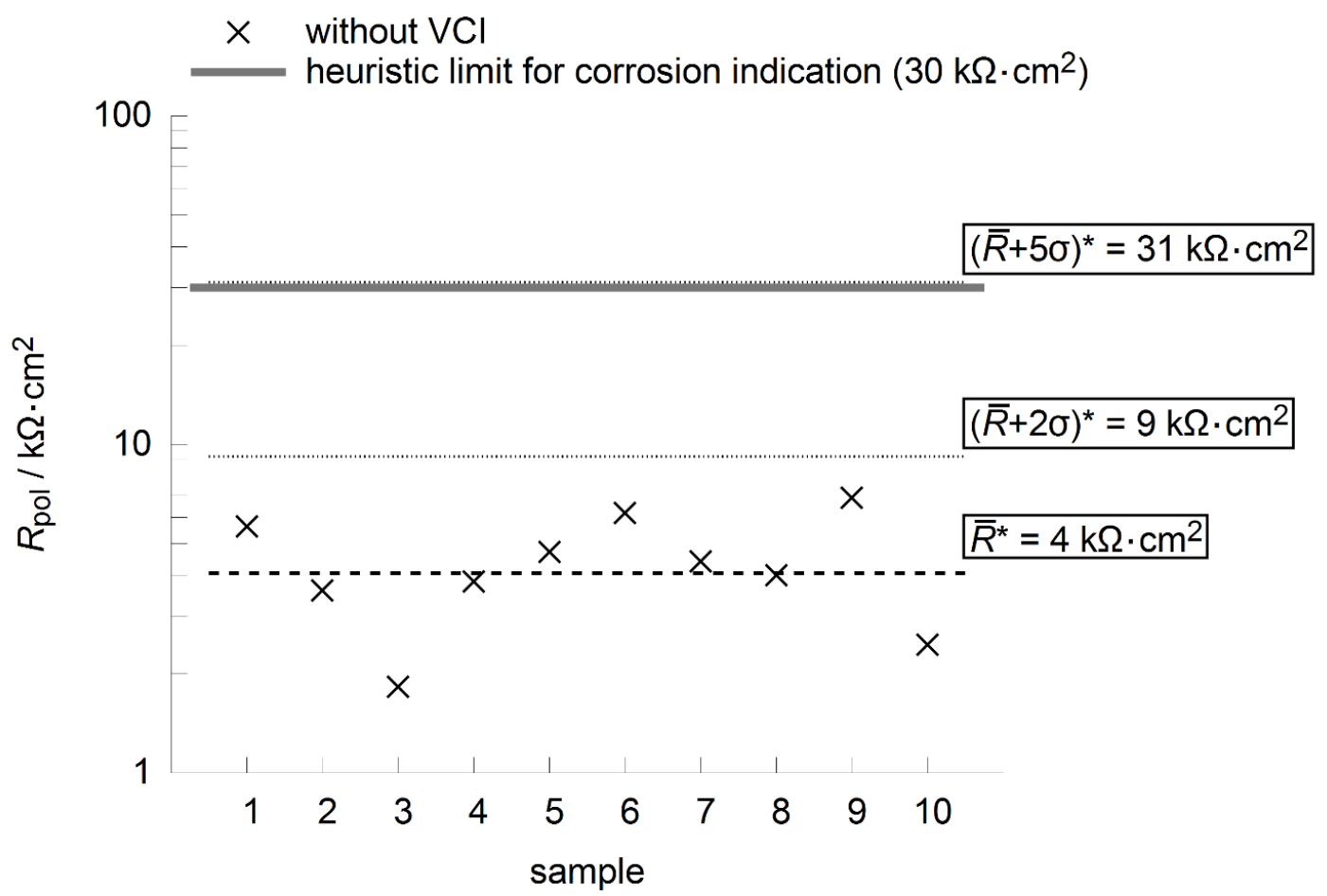

Figure 7. Mean values and $2 \sigma$-intervals of the logarithmized polarization resistance for a sample of 10 independent tests without VCI with respect to the heuristically established limit for corrosion indication of $30 \mathrm{k} \Omega \cdot \mathrm{cm}^{2}$ [17]. The upper value of the $5 \sigma$-interval approximately matches the established limit for corrosion indication. The probability that this value is exceeded by chance in a test without VCI is $<3 \cdot 10^{-5} \%$. Based on the sample, the probability that $R_{\text {pol }}$ by chance exceeds $9 \mathrm{k} \Omega \cdot \mathrm{cm}^{2}$ in a test without VCI is below $2.3 \%$ (upper value of $2 \sigma-$ interval). Therefore, $9 \mathrm{k} \Omega \cdot \mathrm{cm}^{2}$ is chosen as the lower limit for indication of a moderate corrosion protection effect.

The resulting evaluation scheme for the quality assessment of VCI-films with regard to their corrosion protection effect in the test is shown in Table 2. When good corrosion protection $\left(R_{\mathrm{pol}}>30 \mathrm{k} \Omega \cdot \mathrm{cm}^{2}\right)$ is established for a VCI-film in the first run, the test is passed immediately. In case of moderate corrosion protection $\left(9 \mathrm{k} \Omega \cdot \mathrm{cm}^{2}<R_{\text {pol }}<\right.$ $30 \mathrm{k} \Omega \cdot \mathrm{cm}^{2}$ ) in the first run, a second run is mandatory to at least confirm the moderate corrosion protection effect in order to pass the test. In case of an insufficient corrosion protection effect $\left(R_{\mathrm{pol}}<9 \mathrm{k} \Omega \cdot \mathrm{cm}^{2}\right)$ in the first run, the test can be passed only if good corrosion protection is determined in the second run. However, in this case, a confirmation of the result is recommended. For reference, the test shall be conducted in absence of any VCI-film as well as in presence of an appropriate model-VCI at least once a month in a shortened test regime. In these two tests, no and good corrosion protection are expected, 
respectively, and the occurrence of these results confirms the proper function of the test device and the test itself. However, it should be noted that the passivation and corrosion behavior is affected by surface morphology, e.g. roughness and imperfections, but also by preparation-induced changes of the composition [19-23]. Therefore, the limit values may need to be reestablished in the case of changing the sample preparation and, of course, in case of changing the metal itself.

Table 2. Verdict for quality assessment of VCI-films in dependence of the corrosion protection evaluation based on polarization resistance measurement in a first and a second run of the proposed test. When a good corrosion protection is achieved in at least one of the test runs, the quality test is passed. Otherwise, a moderate corrosion protection effect has to be confirmed in a second test run to pass the test.

\begin{tabular}{ccc}
\hline $\begin{array}{c}\text { Corrosion protection } \\
\text { evaluation test \#1 }\end{array}$ & $\begin{array}{c}\text { Corrosion protection } \\
\text { evaluation test \#2 }\end{array}$ & Test verdict \\
\hline good & $/$ & pass \\
$\left(R_{\mathrm{pol}}>30 \mathrm{k} \Omega \cdot \mathrm{cm}^{2}\right)$ & good & pass \\
moderate & moderate & pass \\
$\left(9 \mathrm{k} \Omega \cdot \mathrm{cm}^{2}<R_{\mathrm{pol}} \leq 30 \mathrm{k} \Omega \cdot \mathrm{cm}^{2}\right)$ & insufficient & fail \\
\hline insufficient & good & pass* \\
$\left(R_{\mathrm{pol}} \leq 9 \mathrm{k} \Omega \cdot \mathrm{cm}^{2}\right)$ & moderate & fail \\
& insufficient & fail \\
\hline
\end{tabular}

*confirmation of result recommended

\subsection{Evaluation of VCI-films with the proposed test}

Figure 8 summarizes the obtained values of the polarization resistance for different VCIfilms with respect to the established limits (see section 3.2) in the proposed test. VCI-films I and II show polarization resistance values well above the established limit for a "good corrosion protection" and therefore pass the test. Compared to the results of the jar test with the respective VCI-films, type I and type II congruently show good corrosion protection in both tests. Meanwhile, VCI-film IV is below the established lower limit for "moderate corrosion protection" in 3 of 5 test runs and therefore fails the test. For VCIfilm III, most of the results indicate "good corrosion protection", too. For the VCI-film of type III, which shows rather inconsistent results in the jar test, the deviation of the obtained polarization resistance is also higher. Overall, good to moderate corrosion protection is shown in the proposed test. On the logarithmic scale, the mean value of the polarization resistance for VCI-film III is 4.69 (approx. $50 \mathrm{k} \Omega \cdot \mathrm{cm}^{2}$ ). The lower limit of the $2 \sigma$-interval of the sample with VCI-film III is 4.05 on the logarithmic scale (approx. $11 \mathrm{k} \Omega \cdot \mathrm{cm}^{2}$ ) and thus scarcely above the limit for moderate corrosion protection effect. Also, in contrast to 
the results of the jar test (Table 1), none of the recorded values for VCI-film III indicates insufficient corrosion protection (probability for undershooting the limit of $10 \mathrm{k} \Omega \cdot \mathrm{cm}^{2}$ by chance based on the current sample is below 2.3\%). However, since the jar test evaluation scheme is on a discrete scale, the deviation of the two tests cannot be compared directly.

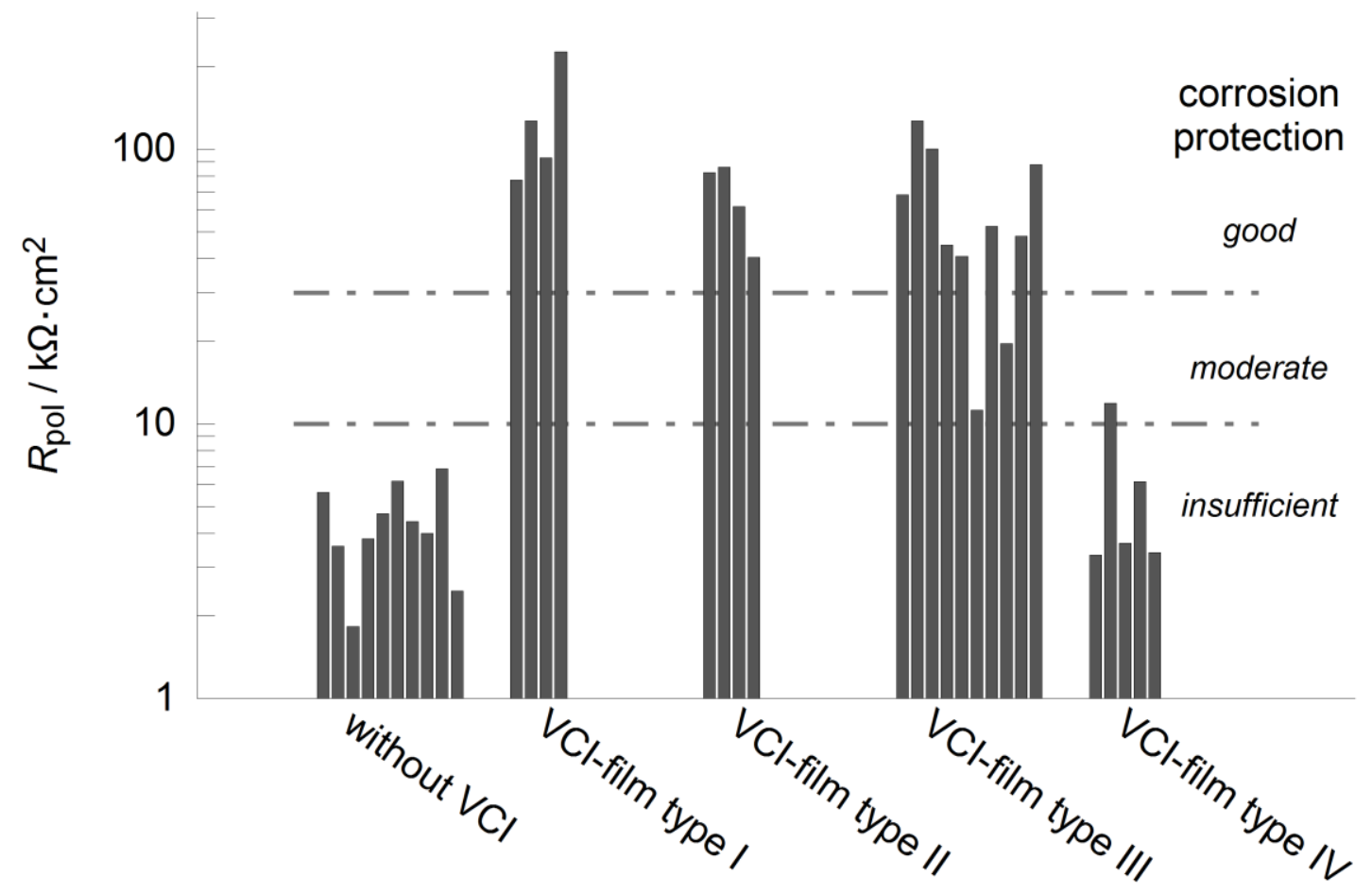

Figure 8. Polarization resistance for independent measurements with the different types of VCI-films with respect to the established limits for indication of good, moderate or insufficient corrosion protection.

Therefore, the distribution of the results of the two tests is shown in Figure 9. Especially the corrosion protection effect of VCI-films III and IV are clearly distinguishable in the proposed test, while the respective results in the jar test are not overly dissimilar. Generally, VCI-films, which show good and moderate corrosion protection in the jar test (marks 3 and 2 on the jar test evaluation scale), show good corrosion protection in the proposed test. In contrast, VCI-films which show weak or no corrosion protection in the jar test (marks 1 and 0 on the jar test evaluation scale) show insufficient corrosion protection in the proposed test. There were only 3 out of the more of 30 independent measurements, in which a moderate corrosion protection effect was established in the proposed test. This is seen as an advantage in terms of quality assessment of VCI-films, because the overall fluctuation of values is less pronounced. This is explained with the state of the metallic sample surface. Preliminary investigations [17] including stopped tests have shown a strong correlation of the measured electrochemical values and the occurrence of corrosion on the surface. With the established test regime 
(Figure 3) with 3 short cycles of climatic stress (stage 2) with low amounts of humidity condensation and evaporation and a final, longer cycle of climatic stress (stage 4) with excessive humidity condensation on the sample surface, it is rather unlikely that the surface is in the stage of early corrosion at the particular point of time, at which the polarization resistance is measured for quality assessment of the respective VCI-films. In presence of an appropriate VCI, the metallic surface is expected to be in the passive state at the point of time of the measurement. Therefore, the measured polarization resistance values will be high. Meanwhile, without VCI or with a VCI product with inappropriate corrosion protection effect, it is very likely that corrosion is initiated already during the 3 short cycles of climatic stress (stage 2) and further progresses during the final climatic stress (stage 4). Therefore, the measured polarization resistance values will be low. This is supported by the overall distribution of the measured values of the polarization resistance. Thus, it can be stated that the occurrence of ambiguous test results cannot be avoided completely, but can be reduced by appropriate design of the test regime.

\section{Conclusions}

A new test regime and a dedicated test device for the evaluation of the corrosion protection effect of VCI-containing products has been proposed. The climatic load on a metallic sample, which is exposed in a chamber alongside a VCI-containing product, is applied reproducibly by active cooling or heating of the sample below or above the dew point of the atmosphere in the chamber with a Peltier element. In contrast to most of the state-ofthe-art tests for VCI, the evaluation of the corrosion protection effect is not based on subjective visual assessment of the metallic sample's surface with regard to the occurrence of corrosion products after the test, but on an objective measurement of the polarization resistance during the test cycle. Based on a statistical analysis of the deviations of the polarization resistance in independently repeated tests without any VCI-material, limit values and an evaluation scheme for qualification of the corrosion protection effect of VCI have been established. The corrosion protection effect of 4 commercially available VCIfilms has been evaluated and compared to the results of the state-of-the-art jar test procedure based on German TL 8135. The analysis of the overall distribution of the results has shown that ambiguous evaluations of the corrosion protection effect are reduced, which makes the proposed test particularly valuable for quality assessment of VCI-containing products within one working day. 

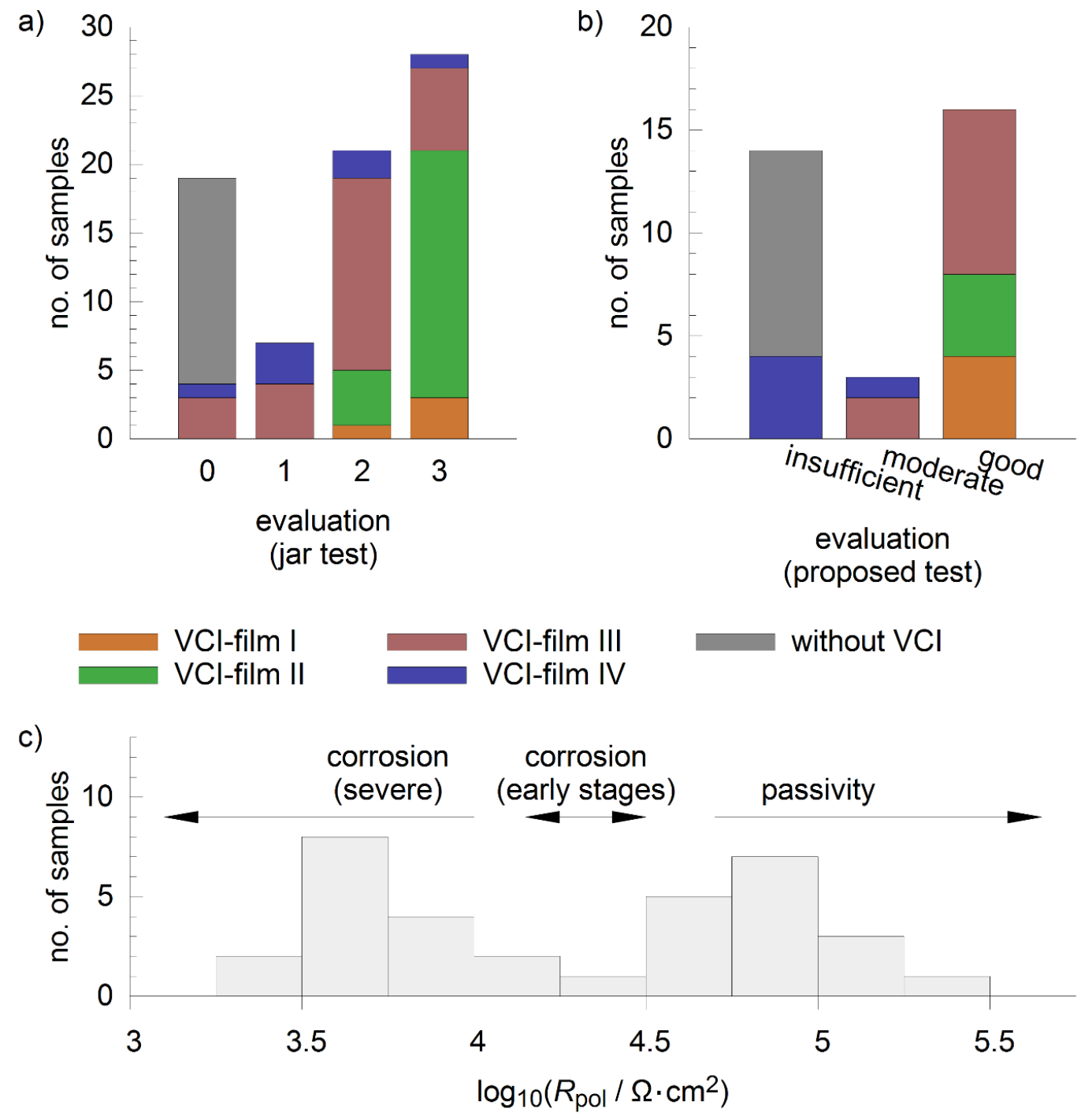

Figure 9. Comparison of the distribution of the obtained results with regard to the evaluation of the corrosion protection effect of the VCI-films in the state-of-the-art jar test (a) and the proposed test based on the measurement of the polarization resistance under climatic stress (b). For independent test repetitions, the deviation in the qualitative test results is smaller in the proposed test, even though VCI-films I and II, which unambiguously possess good corrosion protection properties, are underrepresented in the sample. With regard to the quantitative results of the proposed test, namely the obtained polarization resistance values of all tests (c), there is a predominance of results in two ranges from 3.5 to 4 on the logarithmic scale (approx. $3 \mathrm{k} \Omega \cdot \mathrm{cm}^{2}$ to $10 \mathrm{k} \Omega \cdot \mathrm{cm}^{2}$ ) and from 4.5 to 5 on the logarithmic scale (approx. $30 \mathrm{k} \Omega \cdot \mathrm{cm}^{2}$ to $100 \mathrm{k} \Omega \cdot \mathrm{cm}^{2}$ ). These ranges indicate severe corrosive attack and passivity, respectively. Only few of the measured polarization resistance values are in the range corresponding to earlier stages of corrosion. Also, there are only few samples, which exceed or undershoot polarization resistance values of 5 and 3.5 on the logarithmic scale, respectively $\left(100 \mathrm{k} \Omega \cdot \mathrm{cm}^{2}\right.$ and $\left.3 \mathrm{k} \Omega \cdot \mathrm{cm}^{2}\right)$. 


\section{References}

1. A. Subramanian, M. Natesan, V.S. Muralidharan, K. Baiakrishnan and T. Vasudevan, Corrosion, 2000, 56, no. 2, 144-155. doi: $10.5006 / 1.3280530$

2. Yu.I. Kuznetsov, Int. J. Corros. Scale Inhib., 2015, 4, no. 1, 15-34. doi: 10.17675/2305-6894-2015-4-1-015-034

3. N.N. Andreev and Yu.I. Kuznetsov, Int. J. Corros. Scale Inhib., 2012, 1, no. 1, 16-25. doi: $10.17675 / 2305-6894-2012-1-1-016-025$

4. N.N. Andreev and Yu.I. Kuznetsov, Int. J. Corros. Scale Inhib., 2012, 1, no. 2, 146153. doi: 10.17675/2305-6894-2012-1-2-146-153

5. S. Koehler and G. Reinhard, Int. J. Corros. Scale Inhib., 2014, 3, no. 4, 286-306. doi: 10.17675/2305-6894-2014-3-4-286-306

6. F.A. Ansari, C. Verma, Y.S. Siddiqui, E.E. Ebenso and M.A. Quraishi, Int. J. Corros. Scale Inhib., 2018, 7, no. 2, 126-150. doi: 10.17675/2305-6894-2018-7-2-2

7. B. Valdez, M. Schorr, N. Cheng, E. Beltran and R. Salinas, Corros. Rev., 2018, 36, no. 3, 227-238. doi: 10.1515/corrrev-2017-0102

8. B. Sadlowsky, J. Petzold and S. Karg, $18^{\text {th }}$ IAPRI World Packaging Conference, 2012, 355.

9. K.T. Kim, H.W. Kim, H.Y. Chang, B.T. Lim, H.B. Park and Y.S. Kim, Adv. Mater. Sci. Eng., 2015, 408138, 16 pp. doi: 10.1155/2015/408138

10. Y.J. Tan, S. Bailey and B. Kinsella, Corros. Sci., 1996, 38, no. 9, 1545-1561. doi: 10.1016/0010-938X(96)00047-9

11. K.F. Khaled and S.S. Abdel-Rehim, Arabian J. Chem., 2011, 4, no. 4, 397-402. doi: 10.1016/j.arabjc.2010.07.006

12. A. Popova, E. Sokolova, S. Raicheva and M. Christov, Corros. Sci., 2003, 45, no. 1, 33-58. doi: 10.1016/S0010-938X(02)00072-0

13. H. Boudellioua, Y. Hamlaoui, L. Tifouti and F. Pedraza, Appl. Surf. Sci., 2019, 473, 449-460. doi: $10.1016 /$ j.apsusc.2018.12.164

14. T. Rabizadeh and S.K. Asl, J. Mol. Liq., 2019, 276, 694-704. doi: 10.1016/j.molliq.2018.11.162

15. W. Huang, L. Hu, C. Liu, J. Pan, Y. Tian and K. Cao, Int. J. Electrochem. Sci., 2018, 13, 11273-11285. doi: $10.20964 / 2018.11 .90$

16. A. Popova, A. Vasilev and T. Deligeorgiev, Port. Electrochim. Acta, 2018, 36, no. 6, 423-435. doi: 10.4152/pea.201806423

17. M. Sieber, S. Lautner and F. Faßbender, IOP Conf. Ser.: Mater. Sci. Eng., 2019. doi: 10.1088/1757-899X/480/1/012029

18. L.M. Rymer, M. Sieber, S. Lautner and F. Faßbender, IOP Conf. Ser.: Mater. Sci. Eng., 2019, 480, conference 1. doi: https://doi.org/10.1088/1757-899X/480/1/012028

19. T. Mehner, Correlations between material and surface state and the corrosion susceptibility of metals, PhD-thesis (Technische Universität Chemnitz, Germany), 2017. doi: http://nbn-resolving.de/urn:nbn:de:bsz:ch1-qucosa-225172 
20. S.M. Lee, W.G. Lee, Y.H. Kim and H. Jang, Corros. Sci., 2012, 63, 404-409. doi: 10.1016/j.corsci.2012.06.031

21. A.C. de Oliveira, M.C.L. de Oliveira, C.T. Rios and R.A. Antunes, Corros. Eng., Sci. Technol., 2016, 51, no. 6, 416-428. doi: 10.1080/1478422X.2015.1131493

22. I. Milosev and B. Kapun, Mater. Sci. Eng., C., 2012, 32, no. 5, 1087-1096. doi: 10.1016/j.msec.2011.11.007

23. D. Wallinder, I. Odnevall Wallinder and C. Leygraf, Corrosion, 2003, 59, no. 3, 220227. doi: $\underline{10.5006 / 1.3277554}$ 